\title{
REMARKS ON ONE-COMPONENT INNER FUNCTIONS
}

\author{
Atte Reijonen \\ Tohoku University, Graduate School of Information Sciences \\ Aoba-ku, Sendai 980-8579, Japan; atte.reijonen@uef.fi
}

\begin{abstract}
A one-component inner function $\Theta$ is an inner function whose level set
\end{abstract}

$$
\Omega_{\Theta}(\varepsilon)=\{z \in \mathbf{D}:|\Theta(z)|<\varepsilon\}
$$

is connected for some $\varepsilon \in(0,1)$. We give a sufficient condition for a Blaschke product with zeros in a Stolz domain to be a one-component inner function. Moreover, a sufficient condition is obtained in the case of atomic singular inner functions. We study also derivatives of one-component inner functions in the Hardy and Bergman spaces. For instance, it is shown that, for $0<p<\infty$, the derivative of a one-component inner function $\Theta$ is a member of the Hardy space $H^{p}$ if and only if $\Theta^{\prime \prime}$ belongs to the Bergman space $A_{p-1}^{p}$, or equivalently $\Theta^{\prime} \in A_{p-1}^{2 p}$.

\section{Examples of one-component inner functions}

Let $\mathbf{D}$ be the open unit disc of the complex plane $\mathbf{C}$. A bounded and analytic function in $\mathbf{D}$ is an inner function if it has unimodular radial limits almost everywhere on the boundary $\mathbf{T}$ of $\mathbf{D}$. In this note, we study so-called one-component inner functions [14], which are inner functions $\Theta$ whose level set

$$
\Omega_{\Theta}(\varepsilon)=\{z \in \mathbf{D}:|\Theta(z)|<\varepsilon\}
$$

is connected for some $\varepsilon \in(0,1)$. In particular, Blaschke products in this class are of interest. For a given sequence $\left\{z_{n}\right\} \subset \mathbf{D} \backslash\{0\}$ satisfying $\sum_{n}\left(1-\left|z_{n}\right|\right)<\infty$, the Blaschke product with zeros $\left\{z_{n}\right\}$ is defined by

$$
B(z)=\prod_{n} \frac{\left|z_{n}\right|}{z_{n}} \frac{z_{n}-z}{1-\bar{z}_{n} z}, \quad z \in \mathbf{D} .
$$

Here each zero $z_{n}$ is repeated according to its multiplicity. In addition, we assume that $\left\{z_{n}\right\}$ is ordered by non-decreasing moduli.

Recently several authors have studied one-component inner functions in the context of model spaces and operator theory; see for instance $[6,8,9,10]$. In addition, Aleksandrov's paper [5], which contains several characterizations for one-component inner functions, is worth mentioning. These references do not offer any concrete examples of infinite one-component Blaschke products; even though, reference [5] offers tools for this purpose. In recent paper [13] by Cima and Mortini, one can find some examples. However, all one-component Blaschke products constructed in [13] have some heavy restrictions. Roughly speaking, zeros of all of them are at least uniformly separated. Recall that $\left\{z_{n}\right\} \subset \mathbf{D}$ is called uniformly separated if

$$
\inf _{n \in \mathbf{N}} \prod_{k \neq n}\left|\frac{z_{k}-z_{n}}{1-\bar{z}_{k} z_{n}}\right|>0 \text {. }
$$

https://doi.org/10.5186/aasfm.2019.4434

2010 Mathematics Subject Classification: Primary 30J05; Secondary 30H10.

Key words: Bergman space, Blaschke product, Hardy space, one-component inner function, singular inner function.

This research was supported by Finnish Cultural Foundation. 
As a concrete example, we mention that the Blaschke product with zeros $z_{n}=1-2^{-n}$ for $n \in \mathbf{N}$ is a one-component inner function [13]. In addition, it is a well-known fact that every finite Blaschke product is a one-component inner function.

For $\gamma \geq 1, \xi \in \mathbf{T}$ and $C>0$, we define

$$
R(\gamma, \xi, C)=\left\{z \in \mathbf{D}:|1-\bar{\xi} z|^{\gamma} \leq C(1-|z|)\right\} .
$$

The region $R(1, \xi, C)$ is a Stolz domain with vertex at $\xi$. Note that in the case $\gamma=1$ we have to assume $C>1$. For $\gamma>1, R(\gamma, \xi, C)$ is a tangential approaching region in $\mathbf{D}$, which touches $\mathbf{T}$ at $\xi$. Denote by $\mathcal{R}_{\gamma}$ the family of all Blaschke products whose zeros lie in some $R(\gamma, \xi, C)$ with a fixed $\gamma$. References related to $\mathcal{R}_{\gamma}$ are for instance $[4,11,21]$. With these preparations we are ready to state our first main result.

Theorem 1. Let $B$ be a member of $\mathcal{R}_{1}$ with zeros $\left\{z_{n}\right\}_{n=1}^{\infty}$. If

$$
\liminf _{n \rightarrow \infty} \frac{\sum_{\left|z_{j}\right|>\left|z_{n}\right|}\left(1-\left|z_{j}\right|\right)}{1-\left|z_{n}\right|}>0,
$$

then $B$ is a one-component inner function.

As a consequence of Theorem 1, we obtain the affirmative answer to the following question posed in [13]: Is the Blaschke product $B$ with zeros $z_{n}=1-n^{-2}$ for $n \in \mathbf{N}$ a one-component inner function? Some other examples of one-component inner functions are listed below. All of these examples can be verified by using the fact that condition (1.1) is valid if $\left\{z_{n}\right\}$ is ordered by strictly increasing moduli and

$$
\liminf _{n \rightarrow \infty} \frac{1-\left|z_{n+1}\right|}{1-\left|z_{n}\right|}>0
$$

Example 2. Let $1<\alpha<\infty$ and $B$ be a Blaschke product with zeros

(a) $z_{n}=1-n^{-\alpha}$ for $n \in \mathbf{N}$, or

(b) $z_{n}=1-\frac{1}{n(\log n)^{\alpha}}$ for $n \in \mathbf{N} \backslash\{1\}$, or

(c) $z_{n}=1-\alpha^{-n}$ for $n \in \mathbf{N}$.

Then $B$ is a one-component inner function.

A Blaschke product $B$ is said to be thin if its zeros $\left\{z_{n}\right\}_{n=1}^{\infty}$ satisfy

$$
\lim _{n \rightarrow \infty}\left(1-\left|z_{n}\right|^{2}\right)\left|B^{\prime}\left(z_{n}\right)\right|=1 \text {. }
$$

We interpret that finite Blaschke products are not thin. By [13, Corollary 21], any thin Blaschke product is not a one-component inner function. Using this fact and [12, Proposition 4.3(i)], we can give an example which shows that condition (1.1) in Theorem 1 is essential.

Example 3. Let $B$ be the Blaschke product with zeros $\left\{w_{n}\right\}_{n=1}^{\infty}$ ordered by strictly increasing moduli and satisfying

$$
\frac{1-\left|w_{n+1}\right|}{1-\left|w_{n}\right|} \longrightarrow 0, \quad n \rightarrow \infty
$$

Then, by [12, Proposition 4.3(i)], $B$ is a thin Blaschke product (with uniformly separated zeros). Consequently, for instance, the Blaschke product with zeros $z_{n}=$ $1-2^{-2^{n}}$ for $n \in \mathbf{N}$ is not a one-component inner function. Note that zeros $\left\{z_{n}\right\}$ lie in $R(1,1, C)$ for every $C>1$ but they do not satisfy (1.1). 
Let us recall a classical result of Frostman [17]: The Blaschke product $B$ with zeros $\left\{z_{n}\right\}$ has a unimodular radial limit at $\xi \in \mathbf{T}$ if and only if

$$
\sum_{n} \frac{1-\left|z_{n}\right|}{\left|\xi-z_{n}\right|}<\infty
$$

A Blaschke product is called a Frostman Blaschke product if it has a unimodular radial limit at every point on $\mathbf{T}$. It is a well-known fact that an infinite Frostman Blaschke product cannot be a one-component inner function; see for instance $[5$, Theorem 1.11] or Theorem A in Section 2. Using this fact, we show that any $\mathcal{R}_{\gamma}$ with $\gamma>1$ contains a member which is not a one-component inner function but its zeros $\left\{z_{n}\right\}$ satisfy (1.1). This means that the hypothesis $B \in \mathcal{R}_{1}$ in Theorem 1 is essential.

Example 4. Fix $\gamma>1$ and choose $\alpha=\alpha(\gamma)>1$ such that $\alpha>\frac{\gamma}{\gamma-1}$. Let $\left\{z_{n}\right\}$ be such that

$$
\left|z_{n}\right|=1-n^{-\alpha} \quad \text { and } \quad\left|1-z_{n}\right|=n^{-\alpha / \gamma}, \quad n \in \mathbf{N} .
$$

Since the sequence $\left\{z_{n}\right\}$ is a subset of $R(\gamma, 1,1)$, all points of $\left\{z_{n}\right\}$ lie in D. Moreover, it is clear that $\left\{z_{n}\right\}$ satisfies the Blaschke condition $\sum_{n}\left(1-\left|z_{n}\right|\right)<\infty$ and (1.1) in Theorem 1. Hence the Blaschke product $B$ with zeros $\left\{z_{n}\right\}$ is well-defined. Furthermore,

$$
\sum_{n=1}^{\infty} \frac{1-\left|z_{n}\right|}{\left|1-z_{n}\right|}=\sum_{n=1}^{\infty} n^{\alpha / \gamma-\alpha}<\infty
$$

and thus, $B$ has a unimodular radial limit at 1 by Frostman's result. Since condition (1.2) is trivially valid for every $\xi \in \mathbf{T} \backslash\{1\}, B$ is an infinite Frostman Blaschke product. Consequently, it is not a one-component inner function.

Recall that a singular inner function takes the form

$$
S_{\sigma}(z)=\exp \left(\int_{\mathbf{T}} \frac{z+\xi}{z-\xi} d \sigma(\xi)\right), \quad z \in \mathbf{D},
$$

where $\sigma$ is a positive measure on $\mathbf{T}$, singular with respect to the Lebesgue measure. If the measure $\sigma$ is atomic, then this definition reduces to the form

$$
S(z)=\exp \left(\sum_{n} \gamma_{n} \frac{z+e^{i \theta_{n}}}{z-e^{i \theta_{n}}}\right), \quad z \in \mathbf{D},
$$

where $\theta_{n} \in[0,2 \pi)$ are distinct points and $\gamma_{n}>0$ satisfy $\sum_{n} \gamma_{n}<\infty$. These functions are known as atomic singular inner functions associated with $\left\{e^{i \theta_{n}}\right\}$ and $\left\{\gamma_{n}\right\}$.

An atomic singular inner function associated with a measure having only finitely many mass points is a one-component inner function; see [13, Corollary 17]. In the literature, one cannot find any example of a one-component singular inner function associated with a measure having infinitely many mass points. However, the following result gives a way to construct such functions.

Theorem 5. Let $S$ be the atomic singular inner function associated with $\left\{e^{i \theta_{n}}\right\}_{n=0}^{\infty}$ and $\left\{\gamma_{n}\right\}_{n=0}^{\infty}$. Moreover, assume that the following conditions are valid:

(i) $\theta_{0}=0,\left\{\theta_{n}\right\}_{n=1}^{\infty} \subset(0,1)$ is strictly decreasing and $\lim _{n \rightarrow \infty} \theta_{n}=0$.

(ii) There exists a constant $C=C(S)>0$ such that $\left|\theta_{n-1}-\theta_{n+1}\right| \leq C \gamma_{n}^{2}$ for all sufficiently large $n \in \mathbf{N}$.

Then $S$ is a one-component inner function. 
Next we give a concrete example of a one-component singular inner function. This example is a direct consequence of Theorem 5 .

Example 6. Let $\theta_{0}=0, \theta_{n}=2^{-n}, \gamma_{0}=1$ and $\gamma_{n}=n^{-2}$ for $n \in \mathbf{N}$. Then the atomic singular inner function $S$ associated with $\left\{e^{i \theta_{n}}\right\}_{n=0}^{\infty}$ and $\left\{\gamma_{n}\right\}_{n=0}^{\infty}$ is a onecomponent inner function.

The remainder of this note is organized as follows. Sections 2 and 3 consist of the proofs of Theorems 1 and 5, respectively. In Section 4, we study one-component inner functions whose derivatives belong to the Hardy or Bergman spaces. In particular, we give partial improvements for [1, Theorem 6.2] and [19, Theorem 3.10].

\section{Proof of Theorem 1}

We begin by stating a modification of [5, Theorem 1.11], which is due to [5, p. 2915, Remark 2]. This result offers two practical characterizations for one-component inner functions and plays an important role in the proofs of Theorems 1 and 5 . Before it we recall that the spectrum $\rho(\Theta)$ of an inner function $\Theta$ is the set of all points on $\mathbf{T}$ in which $\Theta$ does not have an analytic continuation. It is a well-known fact that the spectrum of a Blaschke product consists of the accumulation points of zeros. By [18, Chapter 2, Theorem 6.2], the spectrum of a singular inner function $S_{\sigma}$ is the closed support of the associated measure $\sigma$.

Theorem A. Let $\Theta$ be an inner function. Then the following statements are equivalent:

(a) $\Theta$ is a one component inner function.

(b) There exists a constant $C=C(\Theta)>0$ such that

$$
\left|\Theta^{\prime \prime}(\zeta)\right| \leq C\left|\Theta^{\prime}(\zeta)\right|^{2}, \quad \zeta \in \mathbf{T} \backslash \rho(\Theta),
$$

and

$$
\liminf _{r \rightarrow 1^{-}}|\Theta(r \xi)|<1, \quad \xi \in \rho(\Theta) .
$$

(c) There exists a constant $C=C(\Theta)>0$ such that (2.1) holds, the Lebesgue measure of $\rho(\Theta)$ is zero and $\Theta^{\prime}$ is not bounded on any arc $\Gamma \subset \mathbf{T} \backslash \rho(\Theta)$ with $\bar{\Gamma} \cap \rho(\Theta) \neq \emptyset$.

Write $f \lesssim g$ if there exists a constant $C>0$ such that $f \leq C g$, while $f \gtrsim g$ is understood in an analogous manner. If $f \lesssim g$ and $f \gtrsim g$, then the notation $f \asymp g$ is used. With these preparations we are ready to prove Theorem 1.

Proof of Theorem 1. If $B$ is an arbitrary Blaschke product with zeros $\left\{z_{n}\right\}$, then

$$
\frac{B^{\prime}(z)}{B(z)}=\sum_{n=1}^{\infty} \frac{\left|z_{n}\right|^{2}-1}{\left(1-\bar{z}_{n} z\right)\left(z_{n}-z\right)} \quad \text { and } \quad|B(z)| \leq \frac{\left|z_{n}-z\right|}{\left|1-\bar{z}_{n} z\right|} .
$$

Using these estimates, one can easily verify

$$
\left|B^{\prime \prime}(z)\right| \leq \frac{\left|B^{\prime}(z)\right|^{2}}{|B(z)|}+2|B(z)|^{-1} \sum_{n=1}^{\infty} \frac{1-\left|z_{n}\right|^{2}}{\left|1-\bar{z}_{n} z\right|^{3}}, \quad z \in \mathbf{D} .
$$

In particular,

$$
\left|B^{\prime \prime}(\zeta)\right| \leq\left|B^{\prime}(\zeta)\right|^{2}+2 \sum_{n=1}^{\infty} \frac{1-\left|z_{n}\right|^{2}}{\left|1-\bar{z}_{n} \zeta\right|^{3}} \asymp\left|B^{\prime}(\zeta)\right|^{2}+\sum_{n=1}^{\infty} \frac{1-\left|z_{n}\right|^{2}}{\left|z_{n}-\zeta\right|^{3}}
$$


for every $\zeta \in \mathbf{T} \backslash \rho(B)$. Using [4, Theorem 2], we deduce that (2.1) (with $\Theta=B$ ) is valid if

$$
\sum_{n=1}^{\infty} \frac{1-\left|z_{n}\right|^{2}}{\left|z_{n}-\zeta\right|^{3}} \lesssim\left(\sum_{n=1}^{\infty} \frac{1-\left|z_{n}\right|^{2}}{\left|z_{n}-\zeta\right|^{2}}\right)^{2}
$$

holds for every $\zeta \in \mathbf{T} \backslash \rho(B)$.

Assume without loss of generality that zeros $\left\{z_{n}\right\}$ of $B$ lie in a Stolz domain $R(1,1, C)$, and remind that $\left\{z_{n}\right\}$ is ordered by non-decreasing moduli. Then $\rho(B)=$ $\{1\}$, and the functions $f$ and $g$, defined by

$$
f(x)= \begin{cases}1, & 0 \leq x<1 \\ \min _{n \leq x}\left(1-\left|z_{n}\right|\right), & 1 \leq x<\infty\end{cases}
$$

and

$$
g(\theta)=\inf \{x: f(x) \leq \theta\}, \quad 0<\theta \leq 1,
$$

are non-increasing. Since $f(w)=1-\left|z_{n}\right|$ for $n \in \mathbf{N}$ and $n \leq w<n+1$, it is clear that $g:(0,1] \rightarrow \mathbf{N} \cup\{0\}, f(x)>\theta$ for $x<g(\theta)$, and $f(x) \leq \theta$ for $x \geq g(\theta)$. Write $\zeta=e^{i \theta}$, and assume without loss of generality that $\theta>0$ is close enough to zero. Using [4, Lemma 3] together with some standard estimates, we obtain

$$
\begin{aligned}
\left(\sum_{n=1}^{\infty} \frac{1-\left|z_{n}\right|^{2}}{\left|z_{n}-\zeta\right|^{3}}\right)^{1 / 2} & \asymp\left(\sum_{n=1}^{\infty} \frac{1-\left|z_{n}\right|^{2}}{|| z_{n}|-\zeta|^{3}}\right)^{1 / 2} \asymp\left(\sum_{n=1}^{\infty} \frac{1-\left|z_{n}\right|}{\left[\left(1-\left|z_{n}\right|\right)^{2}+\theta^{2}\right]^{3 / 2}}\right)^{1 / 2} \\
& \leq\left(\sum_{n<g(\theta)} f(n)^{-2}+\theta^{-3} \sum_{n \geq g(\theta)} f(n)\right)^{1 / 2} \\
& \leq\left(\sum_{n<g(\theta)} f(n)^{-2}\right)^{1 / 2}+\theta^{-3 / 2}\left(\sum_{n \geq g(\theta)} f(n)\right)^{1 / 2} \\
& \leq \sum_{n<g(\theta)} f(n)^{-1}+\theta^{-3 / 2}\left(\sum_{n \geq g(\theta)} f(n)\right)^{1 / 2} .
\end{aligned}
$$

Applying hypothesis (1.1) and the above-mentioned properties of $f$ and $g$, we find $C=C(B)>0$ such that

$$
\sum_{n \geq g(\theta)} f(n) \geq C f(g(\theta)-1) \geq C \theta .
$$

It follows that

$$
\begin{aligned}
\sum_{n=1}^{\infty} \frac{1-\left|z_{n}\right|^{2}}{\left|z_{n}-\zeta\right|^{2}} & \asymp \sum_{n=1}^{\infty} \frac{1-\left|z_{n}\right|}{\left(1-\left|z_{n}\right|\right)^{2}+\theta^{2}} \\
& \geq \frac{1}{2} \sum_{n<g(\theta)} f(n)^{-1}+\frac{\theta^{-2}}{2} \sum_{n \geq g(\theta)} f(n) \\
& \geq \frac{1}{2} \sum_{n<g(\theta)} f(n)^{-1}+\frac{\sqrt{C} \theta^{-3 / 2}}{2}\left(\sum_{n \geq g(\theta)} f(n)\right)^{1 / 2}
\end{aligned}
$$


Using estimates (2.4) and (2.5), it is easy to see that condition (2.3) is valid for $\zeta \in \mathbf{T} \backslash\{1\}$. Consequently, $B$ satisfies (2.1).

Let $B_{0}$ be the Blaschke product with zeros $\left\{\left|z_{n}\right|\right\}$. It is obvious that $\liminf _{r \rightarrow 1^{-}}$ $\left|B_{0}(r)\right|=0$. Hence, by the deduction above, it is clear that $B_{0}$ satisfies condition (b) in Theorem A, and thus also the other conditions are valid. Since $B_{0}$ satisfies (c) in Theorem A, also $B$ satisfies it. This is due to [4, Lemma 3], which asserts that $\left|B^{\prime}(\xi)\right| \asymp\left|B_{0}^{\prime}(\xi)\right|$ for $\xi \in \mathbf{T} \backslash\{1\}$. Hence $B$ is a one-component inner function by Theorem A. This completes the proof.

\section{Proof of Theorem 5}

Let us prove Theorem 5 .

Proof of Theorem 5. Due to hypothesis (i), the set of mass points $\left\{e^{i \theta_{n}}\right\}_{n=0}^{\infty}$ is closed. Consequently, the spectrum $\rho(S)$ consists of points $\left\{e^{i \theta_{n}}\right\}_{n=0}^{\infty}$. Hence, by [18, Chapter 2, Theorem 6.2], we have

$$
\lim _{r \rightarrow 1^{-}}|S(r \xi)|=0, \quad \xi \in \rho(S) .
$$

This means that $S$ satisfies condition (2.2) (with $\Theta=S$ ) in Theorem A. Consequently, it suffices to show that $S$ fulfills also (2.1).

By a straightforward calculation, one can check that

$$
S^{\prime \prime}(z)=4\left(\sum_{n=0}^{\infty} \frac{\gamma_{n} e^{i \theta_{n}}}{\left(z-e^{i \theta_{n}}\right)^{3}}+\left(\sum_{m=0}^{\infty} \frac{\gamma_{m} e^{i \theta_{m}}}{\left(z-e^{i \theta_{m}}\right)^{2}}\right)^{2}\right) \exp \left(\sum_{k=0}^{\infty} \gamma_{k} \frac{z+e^{i \theta_{k}}}{z-e^{i \theta_{k}}}\right), \quad z \in \mathbf{D} .
$$

Since

$$
\left|S^{\prime}(\zeta)\right|=2 \sum_{n=0}^{\infty} \frac{\gamma_{n}}{\left|\zeta-e^{i \theta_{n}}\right|^{2}}, \quad \zeta \in \mathbf{T} \backslash \rho(S)
$$

by $[4$, Theorem 2], we obtain

$$
\left|S^{\prime \prime}(\zeta)\right| \leq 4 \sum_{n=0}^{\infty} \frac{\gamma_{n}}{\left|\zeta-e^{i \theta_{n}}\right|^{3}}+\left|S^{\prime}(\zeta)\right|^{2}, \quad \zeta \in \mathbf{T} \backslash \rho(S)
$$

Consequently, it suffices to show

$$
\sum_{n=0}^{\infty} \frac{\gamma_{n}}{\left|\zeta-e^{i \theta_{n}}\right|^{3}} \lesssim\left(\sum_{n=0}^{\infty} \frac{\gamma_{n}}{\left|\zeta-e^{i \theta_{n}}\right|^{2}}\right)^{2}, \quad \zeta \in \mathbf{T} \backslash \rho(S)
$$

Assume without loss of generality that $\zeta \in \mathbf{T} \backslash \rho(S)$ is close enough to one, and write $\zeta=e^{i \theta}$. Choose $j=j(\theta, S) \in \mathbf{N} \cup\{0\}$ such that $\left|\theta-\theta_{j}\right|$ is as small as possible. Then standard estimates yield

$$
\begin{aligned}
\left(\sum_{n=0}^{\infty} \frac{\gamma_{n}}{\left|\zeta-e^{i \theta_{n}}\right|^{3}}\right)^{1 / 2} & \asymp\left(\sum_{n=0}^{\infty} \frac{\gamma_{n}}{\left|\theta-\theta_{n}\right|^{3}}\right)^{1 / 2} \\
& \leq\left|\theta-\theta_{j}\right|^{-3 / 2}\left(\sum_{n=0}^{\infty} \gamma_{n}\right)^{1 / 2} \asymp\left|\theta-\theta_{j}\right|^{-3 / 2}
\end{aligned}
$$

and

$$
\sum_{n=0}^{\infty} \frac{\gamma_{n}}{\left|\zeta-e^{i \theta_{n}}\right|^{2}} \asymp \sum_{n=0}^{\infty} \frac{\gamma_{n}}{\left|\theta-\theta_{n}\right|^{2}} \geq \frac{\gamma_{j}}{\left|\theta-\theta_{j}\right|^{2}}
$$


If $\theta<0$, then $j=0$; and hence, (3.1) is a direct consequence of (3.2) and (3.3). Let $\theta>0$. By hypothesis (i), we have $\theta_{j+1}<\theta<\theta_{j-1}$, where $j \in \mathbf{N}$ is large enough. Consequently, hypothesis (ii) gives

$$
\frac{\gamma_{j}}{\left|\theta-\theta_{j}\right|^{2}} \geq \frac{\gamma_{j}}{\left|\theta-\theta_{j}\right|^{3 / 2}\left|\theta_{j-1}-\theta_{j+1}\right|^{1 / 2}} \gtrsim\left|\theta-\theta_{j}\right|^{-3 / 2}
$$

According to this estimate, (3.1) is a consequence of (3.2) and (3.3). Finally the assertion follows from Theorem A.

\section{Derivatives of one-component inner functions in function spaces}

We begin by fixing the notation. Let $\mathcal{H}(\mathbf{D})$ be the space of all analytic functions in $\mathbf{D}$. For $0<p<\infty$, the Hardy space $H^{p}$ consists of those $f \in \mathcal{H}(\mathbf{D})$ such that

$$
\|f\|_{H^{p}}=\sup _{0 \leq r<1} M_{p}(r, f)<\infty, \quad \text { where } \quad M_{p}^{p}(r, f)=\frac{1}{2 \pi} \int_{0}^{2 \pi}\left|f\left(r e^{i \theta}\right)\right|^{p} d \theta .
$$

For $0<p<\infty$ and $-1<\alpha<\infty$, the Bergman space $A_{\alpha}^{p}$ consists of those $f \in \mathcal{H}(\mathbf{D})$ such that

$$
\|f\|_{A_{\alpha}^{p}}^{p}=\int_{\mathbf{D}}|f(z)|^{p}(1-|z|)^{\alpha} d A(z)<\infty
$$

where $d A(z)=d x d y$ is the Lebesgue area measure on $\mathbf{D}$.

By [23, Theorem 5] and [28, Lemma 1.4], we have

$$
\left\{f: f^{\prime} \in A_{p-1}^{p}\right\} \subset H^{p}, \quad 0<p \leq 2 .
$$

and

$$
H^{p} \subset\left\{f: f^{\prime} \in A_{p-1}^{p}\right\}, \quad 2 \leq p<\infty .
$$

It is clear that $\left\{f: f^{\prime} \in A_{1}^{2}\right\}=H^{2}$, while otherwise the inclusions are strict. For instance, an example showing the strictness of inclusions (4.1) and (4.2) can be given by using gap series; see details in [7]. Nevertheless, we have the following result, which is essentially a consequence of [1, Theorem 6.2] and [19, Theorem 3.10].

Theorem 7. Let $\frac{1}{2}<p<\infty$ and $\Theta$ be an inner function. Then the following statements are equivalent:

(a) $\Theta^{\prime} \in H^{p}$,

(b) $\Theta^{\prime} \in A_{p-1}^{2 p}$,

(c) $\Theta^{\prime \prime} \in A_{p-1}^{p}$.

Before the proof of Theorem 7, we note that, for $f \in \mathcal{H}(\mathbf{D}), n \in \mathbf{N}$ and $0<p<$ $\infty$, we have $M_{p}\left(r, f^{(n)}\right) \asymp M_{p}\left(r, D^{n} f\right)$ with comparison constants independent of $r$ [16]. Here $D^{n}$ is the fractional derivative of order $n$. This fact is exploited when we apply some results in the literature.

Proof. The equivalence (a) $\Leftrightarrow$ (c) is a consequence of [19, Theorem 3.10]. For $\frac{1}{2}<$ $p<1$, the equivalence (a) $\Leftrightarrow$ (b) can be verified, for instance, using [1, Theorem 6.2] together with [26, Corollary 7]. It is a well-known fact the only inner functions whose derivative belongs to $H^{p}$ for some $p \geq 1$ are finite Blaschke products. Using this fact together with $[22$, Theorem $7(\mathrm{c})]$ and the equivalence $(\mathrm{a}) \Leftrightarrow(\mathrm{c})$, it is easy to deduce that an inner function $\Theta$ is a finite Blaschke product if it satisfies any of conditions (a)-(c) for some $p \geq 1$. In addition, it is clear that every finite Blaschke product $\Theta$ satisfies conditions (a)-(c) for all $p>0$. Finally the assertion follows by combining the above-mentioned facts. 
By [3, Lemma 2], there exists a Blaschke product $B$ such that $B^{\prime} \in A_{-1 / 2}^{1} \backslash H^{1 / 2}$. This means that, for $p=\frac{1}{2}$, condition (b) in Theorem 7 does not always imply (a). Nevertheless, it is an open question whether the equivalence (a) $\Leftrightarrow$ (c) is valid also for $0<p \leq \frac{1}{2}$. This question was earlier posed in [27]. The next result shows that the statement of Theorem 7 is valid for all $p>0$ if $\Theta$ is a one-component inner function. Consequently, we obtain a partial answer to the question.

Theorem 8. Let $0<p<\infty$ and $\Theta$ be a one-component inner function. Then conditions (a)-(c) in Theorem 7 are equivalent.

Next we recall [5, Theorem 1.9], which consists of a strengthened Schwarz-Pick lemma for one-component inner functions. This result plays a key role in the proof of Theorem 8.

Theorem B. Let $n \in \mathbf{N}$ and $\Theta$ be a one-component inner function. Then there exists $C=C(n, \Theta)>0$ such that

$$
\left|\Theta^{(n)}(z)\right| \leq C\left(\frac{1-|\Theta(z)|}{1-|z|}\right)^{n}
$$

for all $z \in \mathbf{D}$.

For the proof of Theorem 8, we need also a generalization of [1, Theorem 6.1].

Lemma 9. Let $0<p<1,-1<\alpha<\infty$ and $\Theta$ be an inner function. Then there exists $C=C(p, \alpha)>0$ such that

$$
\int_{0}^{1}\left|\Theta^{\prime}\left(r e^{i \theta}\right)\right|^{p+\alpha+1}(1-r)^{\alpha} d r \leq C\left|\Theta^{\prime}\left(e^{i \theta}\right)\right|^{p}, \quad e^{i \theta} \in \mathbf{T} \backslash \rho(\Theta) .
$$

In particular, $\left\|\Theta^{\prime}\right\|_{A_{\alpha}^{p+\alpha+1}}^{p+\alpha+1} \leq 2 \pi C\left\|\Theta^{\prime}\right\|_{H^{p}}^{p}$.

Proof. Let $e^{i \theta} \in \mathbf{T} \backslash \rho(\Theta)$. By [1, Lemma 6.1], we know that $\left|\Theta^{\prime}\left(r e^{i \theta}\right)\right| \leq 4\left|\Theta^{\prime}\left(e^{i \theta}\right)\right|$ for all $r \in[0,1)$. Using this fact together with the Schwarz-Pick lemma, we obtain

$$
\begin{aligned}
\int_{0}^{1}\left|\Theta^{\prime}\left(r e^{i \theta}\right)\right|^{p+\alpha+1}(1-r)^{\alpha} d r & \leq \int_{0}^{x}(1-r)^{-p-1} d r+\left(4\left|\Theta^{\prime}\left(e^{i \theta}\right)\right|\right)^{p+\alpha+1} \int_{x}^{1}(1-r)^{\alpha} d r \\
& \lesssim(1-x)^{-p}-1+(1-x)^{\alpha+1}\left|\Theta^{\prime}\left(e^{i \theta}\right)\right|^{p+\alpha+1}
\end{aligned}
$$

for every $x \in[0,1]$. Now it suffices to show that

$$
(1-x)^{-p}-1+(1-x)^{\alpha+1}\left|\Theta^{\prime}\left(e^{i \theta}\right)\right|^{p+\alpha+1} \lesssim\left|\Theta^{\prime}\left(e^{i \theta}\right)\right|^{p}
$$

for some $x$. If $\left|\Theta^{\prime}\left(e^{i \theta}\right)\right| \leq 1$, then this true for $x=0$. In the case where $\left|\Theta^{\prime}\left(e^{i \theta}\right)\right|>1$, the choice $x=1-1 /\left|\Theta^{\prime}\left(e^{i \theta}\right)\right|$ implies (4.4). Since the last assertion is a direct consequence of the first assertion, Hardy's convexity and the mean convergence theorems [15], the proof is complete.

Now we are ready to prove Theorem 8 .

Proof of Theorem 8. By Theorem 7, we may assume $0<p<1$ (or even $p \leq \frac{1}{2}$ ). Using Theorem B with $n=2$, [2, Theorem 6] and Lemma 9 with $\alpha=p-1$, we obtain

$$
\left\|\Theta^{\prime \prime}\right\|_{A_{p-1}^{p}}^{p} \lesssim \int_{\mathbf{D}}\left(\frac{1-|\Theta(z)|}{1-|z|}\right)^{2 p}(1-|z|)^{p-1} d A(z) \asymp\left\|\Theta^{\prime}\right\|_{A_{p-1}^{2 p}}^{2 p} \lesssim\left\|\Theta^{\prime}\right\|_{H^{p}}^{p} .
$$

The assertion follows from (4.1) and (4.5). 
It is a well-known fact that, for $0<p<\infty$ and $-1<\alpha<\infty$, the Bergman space $A_{\alpha}^{p}$ coincides with $\left\{f: f^{\prime} \in A_{\alpha+p}^{p}\right\}$ [16]. Using this result, it is easy to generalize condition (c) in Theorem 8 to the form $\Theta^{(n)} \in A_{p(n-1)-1}^{p}$ for any/every $n \in \mathbf{N} \backslash\{1\}$. For $0<p<1$, we can show this also by modifying the proof of Theorem 8; and as a substitute of this process we obtain an alternative version of Theorem 8 .

Theorem 10. Let $0<p<\infty$ and $\Theta$ be a one-component inner function. Then the following statements are equivalent:

(a) $\Theta^{\prime} \in H^{p}$,

(b) $\Theta^{\prime} \in A_{\alpha}^{p+\alpha+1}$ for some $\alpha \in(-1, \infty)$,

(c) $\Theta^{\prime} \in A_{\alpha}^{p+\alpha+1}$ for every $\alpha \in(-1, \infty)$.

Proof. By the proof of Theorem 7, we know that, for $1 \leq p<\infty, \Theta$ satisfies any/all of conditions (a)-(c) if and only if it is a finite Blaschke product. Hence we may assume $0<p<1$. Moreover, let $-1<\alpha<\infty$ and $n \in \mathbf{N} \backslash\{1\}$. Then [16, Theorem 3], Theorem B, [2, Theorem 6], the Schwarz-Pick lemma and Lemma 9 yield

$$
\begin{aligned}
\left\|\Theta^{\prime}\right\|_{H^{p}}^{p} & \lesssim\left\|\Theta^{(n)}\right\|_{A_{p(n-1)-1}^{p}}^{p} \lesssim \int_{\mathbf{D}}\left(\frac{1-|\Theta(z)|}{1-|z|}\right)^{n p}(1-|z|)^{p(n-1)-1} d A(z) \\
& \asymp\left\|\Theta^{\prime}\right\|_{A_{p(n-1)-1}^{n p}}^{n p} \leq\left\|\Theta^{\prime}\right\|_{A_{\alpha}^{p+\alpha+1}}^{p+\alpha+1} \lesssim\left\|\Theta^{\prime}\right\|_{H^{p}}^{p}
\end{aligned}
$$

when $p+\alpha+1 \leq n p$. Since we may choose $n=n(p, \alpha)$ such that $n \geq(p+\alpha+1) / p$, the assertion follows from (4.6).

Note that, applying Theorem 10 and [27, Theorem 3], we can give several characterizations for one-component inner functions $\Theta$ whose derivative belongs to $H^{p}$ for some $p \in(0,1)$. By [27, Corollary 4], these characterizations for $p \in\left(\frac{1}{2}, 1\right)$ are valid even if $\Theta$ would be an arbitrary inner function. Next we show a counterpart of Theorem 10 for all members of $\mathcal{R}_{1}$.

Corollary 11. Let $0<p<\infty$ and $B \in \mathcal{R}_{1}$. Then the following statements are equivalent:

(a) $B^{\prime} \in H^{p}$,

(b) $B^{\prime} \in A_{\alpha}^{p+\alpha+1}$ for some $\alpha \in(-1, \infty)$,

(c) $B^{\prime} \in A_{\alpha}^{p+\alpha+1}$ for every $\alpha \in(-1, \infty)$.

Proof. Assume without loss of generality that $0<p<1$ and zeros $\left\{w_{n}\right\}$ of $B$ lie in a Stolz domain $R(1,1, C)$. Let $B_{0}$ be the Blaschke product with zeros $x_{n}=1-2^{-n}$ for $n \in \mathbf{N}$, write $\Theta=B B_{0}$ and $\left\{z_{n}\right\}=\left\{w_{n}\right\} \cup\left\{x_{n}\right\}$, where $\left\{z_{n}\right\}$ is ordered by nondecreasing moduli. Then, for each $n \in \mathbf{N}$, there exists $k_{n} \in \mathbf{N}$ such that

$$
\left|x_{k_{n}}\right| \leq\left|z_{n}\right|<\left|x_{k_{n}+1}\right|
$$

It follows that

$$
\frac{\sum_{\left|z_{j}\right|>\left|z_{n}\right|}\left(1-\left|z_{j}\right|\right)}{1-\left|z_{n}\right|} \geq \frac{1-\left|x_{k_{n}+1}\right|}{1-\left|x_{k_{n}}\right|}=\frac{1}{2} .
$$

Consequently, $\Theta$ is a one-component inner function by Theorem 1.

Let $-1<\alpha<\infty$. By [4, Theorem 5] and a simple modification of [25, Corollary 2.5] based on [2, Theorem 6], we know that

$$
\Theta^{\prime} \in H^{p} \quad \Leftrightarrow \quad B^{\prime} \in H^{p} \quad \text { and } \quad B_{0}^{\prime} \in H^{p}
$$


and

$$
\Theta^{\prime} \in A_{\alpha}^{p+\alpha+1} \Leftrightarrow B^{\prime} \in A_{\alpha}^{p+\alpha+1} \quad \text { and } \quad B_{0}^{\prime} \in A_{\alpha}^{p+\alpha+1} .
$$

In addition, [4, Theorem 7] and Lemma 9 imply $B_{0}^{\prime} \in H^{p} \cap A_{\alpha}^{p+\alpha+1}$. Finally, the assertion follows by using Theorem 10 together with the above-mentioned facts.

Since the derivative of an arbitrary $B \in \mathcal{R}_{1}$ belongs to $H^{p} \cap A_{\alpha}^{p+\alpha+1}$ for every $p \in\left(0, \frac{1}{2}\right)$ and $\alpha \in(-1, \infty)$ by [20, Theorem 2.3] and Lemma 9 , the statement of Corollary 11 for $p \neq \frac{1}{2}$ does not come as a surprise. However, the case $p=\frac{1}{2}$ is interesting because it is not easy to find an alternative way to prove this result.

Recall that $f \in \mathcal{H}(\mathbf{D})$ belongs to the Nevalinna class $\mathcal{N}$ if

$$
\sup _{0 \leq r<1} \int_{0}^{2 \pi} \log ^{+}\left|f\left(r e^{i \theta}\right)\right| d \theta<\infty,
$$

where $\log ^{+} 0=0$ and $\log ^{+} x=\max \{0, \log x\}$ for $0<x<\infty$. As a consequence of Theorem B, we can also give sufficient conditions for higher order derivatives of one-component inner functions to be in the Hardy space $H^{p}$ or Nevanlinna class $\mathcal{N}$.

Corollary 12. Let $0<p<\infty, n \in \mathbf{N}$ and $\Theta$ be a one-component inner function. Then the following statements are valid:

(a) If $\Theta^{\prime} \in H^{p}$, then $\Theta^{(n)} \in H^{p / n}$.

(b) If $\Theta^{\prime} \in \mathcal{N}$, then $\Theta^{(n)} \in \mathcal{N}$.

Proof. As a consequence of Theorem B [5], we find $C=C(n, \Theta)$ such that

$$
\left|\Theta^{(n)}(\xi)\right| \leq C\left|\Theta^{\prime}(\xi)\right|^{n}, \quad \xi \in \mathbf{T} \backslash \rho(\Theta) .
$$

Since the spectrum $\rho(\Theta)$ has a Lebesgue measure zero, inequality (4.7), Hardy's convexity and the mean convergence theorems yield

$$
\left\|\Theta^{(n)}\right\|_{H^{p / n}}^{p / n}=\frac{1}{2 \pi} \int_{0}^{2 \pi}\left|\Theta^{(n)}\left(e^{i \theta}\right)\right|^{p / n} d \theta \lesssim \frac{1}{2 \pi} \int_{0}^{2 \pi}\left|\Theta^{\prime}\left(e^{i \theta}\right)\right|^{p} d \theta=\left\|\Theta^{\prime}\right\|_{H^{p}}^{p} .
$$

Hence assertion (a) is proved. Since case (b) can be verified in a similar manner, the proof is complete.

We close this note with two results regarding certain one-component singular inner functions.

Corollary 13. Let $0<p<\infty$ and $S$ be the one-component atomic singular inner function associated with $\left\{e^{i \theta_{n}}\right\}$ and $\left\{\gamma_{n}\right\} \in l^{1 / 2}$. Then $S$ satisfies any/all of conditions (a)-(c) in Theorem 7 if and only if $p<\frac{1}{2}$.

Proof. By [26, Theorem 3], for $\frac{1}{4} \leq p<\infty$, the derivative of $S$ belongs to $A_{p-1}^{2 p}$ if and only if $p<\frac{1}{2}$. Since $H^{p_{1}} \subset H^{p_{2}}$ for $0<p_{2} \leq p_{1}<\infty$, the assertion follows from this result and Theorem 8.

The following result shows that Corollary 12(a) is sharp.

Corollary 14. Let $0<p<\infty, m \in \mathbf{N}$ and $S$ be the one-component atomic singular inner function associated with $\left\{e^{i \theta_{n}}\right\}$ and $\left\{\gamma_{n}\right\} \in l^{1 / 2}$. Moreover, assume that there exist an index $j=j(S)$ and $\varepsilon=\varepsilon(j)>0$ such that $\left|\theta_{j}-\theta_{n}\right|>\varepsilon$ for all $n \neq j$. Then $S^{(m)} \in H^{p}$ if and only if $p<\frac{1}{2 m}$.

Proof. By Corollary 13, $S^{\prime} \in H^{m p}$ if and only if $p<\frac{1}{2 m}$. Consequently, Corollary 12(a) implies $S^{(m)} \in H^{p}$ for $p<\frac{1}{2 m}$. Hence it suffices to show that $S^{(m)} \in H^{p}$ only if $p<\frac{1}{2 m}$. 
Fix $j=j(S)$ to be the smallest index such that $\left|\theta_{j}-\theta_{n}\right|>\varepsilon$ for all $n \neq j$ and some $\varepsilon=\varepsilon(j)>0$. Let us represent $S$ in the form $S=S_{1} S_{2}$, where

$$
S_{1}(z)=\exp \left(\gamma_{j} \frac{z+e^{i \theta_{j}}}{z-e^{i \theta_{j}}}\right), \quad z \in \mathbf{D},
$$

and $S_{2}=S / S_{1}$. Using this factorization, we obtain

$$
\left|S^{(m)}\left(e^{i \theta}\right)\right|=\left|\sum_{k=0}^{m}\left(\begin{array}{c}
m \\
k
\end{array}\right) S_{1}^{(m-k)}\left(e^{i \theta}\right) S_{2}^{(k)}\left(e^{i \theta}\right)\right| \asymp\left|S_{1}^{(m)}\left(e^{i \theta}\right) S_{2}\left(e^{i \theta}\right)\right|=\left|S_{1}^{(m)}\left(e^{i \theta}\right)\right|
$$

when $\theta$ (which is not $\theta_{j}$ ) is close enough to $\theta_{j}$ depending on $S$ and $m$. Consequently, we find a sufficiently small $\alpha=\alpha(p, S, m)>0$ such that

$$
\int_{0}^{2 \pi}\left|S_{1}^{(m)}\left(e^{i \theta}\right)\right|^{p} d \theta \asymp \int_{\theta_{j}-\alpha}^{\theta_{j}+\alpha}\left|S_{1}^{(m)}\left(e^{i \theta}\right)\right|^{p} d \theta \lesssim \int_{0}^{2 \pi}\left|S^{(m)}\left(e^{i \theta}\right)\right|^{p} d \theta,
$$

where the comparison constants depend only on $p, S$ and $m$. It follows that $S^{(m)} \in H^{p}$ only if $S_{1}^{(m)} \in H^{p}$. Moreover, a simple modification of the main result of [24] shows that $S_{1}^{(m)} \in H^{p}$ if and only if $p<\frac{1}{2 m}$. Combining these facts, we deduce that $S^{(m)} \in H^{p}$ (if and) only if $p<\frac{1}{2 m}$. This completes the proof.

Acknowledgements. The author thanks Toshiyuki Sugawa for valuable comments, and the referees for careful reading of the manuscript.

\section{References}

[1] Ahern, P.: The mean modulus and the derivative of an inner function. - Indiana Univ. Math. J. 28:2, 1979, 311-347.

[2] Ahern, P.: The Poisson integral of a singular measure. - Canad. J. Math. 35:4, 1983, 735-749.

[3] Ahern, P.R., and D. N. Clark: On inner functions with $B^{p}$ derivative. - Michigan Math. J. 23:2, 1976, 107-118.

[4] Ahern, P.R., and D. N. Clark: On inner functions with $H^{p}$ derivative. - Michigan Math. J. 21, 1974, 115-127.

[5] Aleksandrov, A. B.: Embedding theorems for coinvariant subspaces of the shift operator. II. - Zap. Nauchn. Sem. S.-Peterburg. Otdel. Mat. Inst. Steklov. (POMI) 262, 1999, Issled. po Lineǐn. Oper. i Teor. Funkts. 27, 5-48, 231; English transl. in J. Math. Sci. (New York) 110:5, 2002, 2907-2929.

[6] Aleman, A., Y. Lyubarskit, E. Malinnikova, and K.-M. Perfekt: Trace ideal criteria for embeddings and composition operators on model spaces. - J. Funct. Anal. 270:3, 2016, 861-883.

[7] Baernstein, A., D. Girela, and J.A. PelÁez: Univalent functions, Hardy spaces and spaces of Dirichlet type. - Illinois J. Math. 48:3, 2004, 837-859.

[8] Baranov, A., R. Bessonov, and V. Kapustin: Symbols of truncated Toeplitz operators. J. Funct. Anal. 261:12, 2011, 3437-3456.

[9] Bessonov, R. V.: Duality theorems for coinvariant subspaces of $H^{1}$. - Adv. Math. 271, 2015, 62-90.

[10] Bessonov, R. V.: Fredholmness and compactness of truncated Toeplitz and Hankel operators. - Integral Equations Operator Theory 82:4, 2015, 451-467.

[11] Cargo, G. T.: Angular and tangential limits of Blaschke products and their successive derivatives. - Canad. J. Math. 14, 1962, 334-348. 
[12] Chalendar, I., E. Fricain, and D. Timotin: Functional models and asymptotically orthonormal sequences. - Ann. Inst. Fourier (Grenoble) 53:5, 2003, 1527-1549.

[13] Cima, J., and R. Mortini: One-component inner functions. - Complex Anal. Synerg. 3:1, 2017, Paper No. 2, 1-15.

[14] Cohn, B.: Carleson measures for functions orthogonal to invariant subspaces. - Pacific J. Math. 103:2, 1982, 347-364.

[15] Duren, P.: Theory of $H^{p}$ spaces. - Academic Press, New York-London, 1970.

[16] FLETt, T. M.: The dual of an inequality of Hardy and Littlewood and some related inequalities. - J. Math. Anal. Appl. 38, 1972, 746-765.

[17] Frostman, O.: Sur les produits de Blaschke. - Kungl. Fysiografiska Sällskapets i Lund Förhandlingar 12:15, 1942, 169-182.

[18] Garnett, J.: Bounded analytic functions. Revised 1st edition. - Springer, New York, 2007.

[19] Girela, D., C. González, and M. Jevtić: Inner functions in Lipschitz, Besov, and Sobolev spaces. - Abstr. Appl. Anal. 2011, 2011, Art. ID 626254, 1-26.

[20] Girela, D., J. A. Peláez, and D. Vukotić: Integrability of the derivative of a Blaschke product. - Proc. Edinb. Math. Soc. (2) 50:3, 2007, 673-687.

[21] Girela, D., J.A. Peláez, and D. Vukotić: Interpolating Blaschke products: Stolz and tangential approach regions. - Constr. Approx. 27:2, 2008, 203-216.

[22] Gluchoff, A.: On inner functions with derivative in Bergman spaces. - Illinois J. Math. 31:3, $1987,518-528$.

[23] Littlewood, J. E., and R. E. A. C. Paley: Theorems on Fourier series and power series (II). - Proc. London Math. Soc. (2) 42:1, 1936, 52-89.

[24] Mateljević, M., and M. Pavlović: On the integral means of derivatives of the atomic function. - Proc. Amer. Math. Soc. 86:3, 1982, 455-458.

[25] Pérez-GonzÁlez, F., and J. Rättyä: Inner functions in the Möbius invariant Besov-type spaces. - Proc. Edinburgh Math. Soc. 52, 2009, 751-770.

[26] Pérez-González, F., J. RÄttyä, and A. Reijonen: Derivatives of inner functions in Bergman spaces induced by doubling weights. - Ann. Acad. Sci. Fenn. Math. 42:2, 2017, 735753.

[27] Reijonen, A., and T. Sugawa: Characterizations for inner functions in certain function spaces. - Complex Anal. Oper. Theory, 2018, 1-19, https://doi.org/10.1007/s11785-018-08639.

[28] Vinogradov, S. A.: Multiplication and division in the space of analytic functions with an area-integrable derivative, and in some related spaces. - Zap. Nauchn. Sem. S.-Peterburg. Otdel. Mat. Inst. Steklov. (POMI) 222, 1995 (in Russian), Issled. po Linein. Oper. i Teor. Funktsii 23, 45-77; English transl. in J. Math. Sci. (New York) 87, 1997, 3806-3827. 